\title{
Current update on herbal sources of antithrombotic activity-a comprehensive review
}

\author{
Bhavani Subramani* (iD and P. Sathiyarajeswaran
}

\begin{abstract}
Background: Herbs are commonly used to treat cardiovascular diseases in various traditional medicine. On the other hand, herb-drug interactions are most commonly encountered with conventional antiplatelet and anticoagulant drug prescriptions. This review presents a compilation of plants investigated for antiplatelet and anticoagulation recently and enumerates their possible lead compounds responsible for its action for paving further drug discovery and knowledge update.

Main body of the abstract: Information about the herbs was withdrawn from the PubMed database of the previous 5 years. We also hand-searched the bibliography of relevant articles for the acquisition of additional information. About 72 herbal sources were identified with the effect of antiplatelet activity, antithrombotic activity, and anticoagulant activity. Bioactive compounds and various secondary metabolites responsible for it, such as alkaloids, saponins, flavonoids, coumarins, polyphenols, furan derivatives, iridoid glycosides, sesquiterpenes, aporphine compounds, were reported.
\end{abstract}

Conclusion: Newer pharmacological moieties are needed to prevent or reduce the adverse effects of current antithrombotic agents and to improve the safety of patients and cost-effectiveness.

Keywords: Antiplatelet, Antithrombotic, Anticoagulant, Herbal medicine, Phytochemicals, Secondary metabolites, Alkaloids, Saponins, Flavonoids, Coumarins

\section{Background}

Cardiovascular disease (CVD) due to thrombosis comprises coronary artery disease (CAD), stroke, hypertension, peripheral arterial disease (PAD), venousthrombo-embolic disease (VTE) [1]. As per the National Health and Nutrition Examination Survey (NHANES) 2013-2016, the prevalence of Coronary heart disease (CHD) in the USA was estimated as 18.2 million in $>20$ years of age with more risk among males than females, whereas the prevalence of ischaemic stroke was 67.6 million and that of hemorrhagic stroke was 15.3 million [2]. CVD and stroke accounted for $14 \%$ of the total expenditure

*Correspondence: msbhavani@hotmail.com

Siddha Central Research Institute (SCRI), Central Council for Research

in Siddha (CCRS), Arumbakkam, Chennai, Tamilnadu 600106, India in 2014-2015, more than any diagnostic group results in immense health and economic burden in the USA globally. The AHA's 2020 Impact Goals are to improve the cardiovascular health of all Americans by $20 \%$ while reducing deaths attributable to CVD and stroke by $20 \%$ [1].

Currently, witnessing an unprecedented pandemic, the coronavirus disease 2019 (COVID-19), caused by severe acute respiratory syndrome coronavirus 2 (SARS $\mathrm{Co}-\mathrm{V}-2$ ), associated with a significant risk of thromboembolic complications due to hypercoagulability state of blood which is called as Covid-19 associated coagulopathy (CAC) [3]. Though prophylaxis anti-coagulants were administered, the incidence of VTE complications was reported in twothirds of ICU cases [4] and developed life-threatening thrombotic complications followed by Acute respiratory distress syndrome (ARDS) [5]. Venous thromboembolism 
(VTE), a major cardiovascular complication, was observed in about more than $20 \%$ of critically ill COVID19 cases, particularly among critically ill viral pneumonia patients [4]. Histologically, significant thrombosis in small blood vessels and micro-vasculature of pulmonary and extra-pulmonary organs have been confirmed [6], widespread prevalence of deep vein thrombosis and pulmonary embolism, as well as microthrombi in the small pulmonary vessels in autopsy findings [7]. Several hypotheses on the mechanism of thrombosis in Covid-19 have been proposed and remain unclear.

\section{Antiplatelets and anti-coagulants}

Thrombosis can be classified as arterial thrombosis and venous thrombosis although overlaps may be present. In general, pharmacologically two classes of drugs are used to prevent blood clots such as antiplatelets and anticoagulants [8]. Antiplatelets act by inhibition of platelet adhesion and activation and aggregation of thrombosis [9]. Thrombosis refers to the formation of platelet or fibrin aggregation in the lumen of the blood vessels or heart [10]. Anticoagulants prevent blood clot formation by interfering with proteins responsible for blood clotting or clotting factors [8]. Hypercoagulability is the state of increased tendency to the formation of thrombosis also triggering intracellular signalling for inflammation [10]. The use of antithrombotic medications remains the mainstay of treatment in cardiovascular and cerebrovascular disorders. Aspirin and clopidogrel were the commonly administered antiplatelet drugs to reduce recurrent ischaemic events in CAD and ischaemic stroke. Oral anticoagulants are prescribed for primary prevention and secondary prevention of venous thromboembolic disease [11] and as the best option in the prevention of stroke due to cardio-embolism in atrial fibrillation [12].

\section{Adverse drug reaction due to conventional antithrombotic drug regimen}

Aspirin is prone to cause gastrointestinal side effects, hypersensitivity, hypo-responsiveness in some, and bleeding episodes [13]. Low-dose aspirin is commonly used as primary and secondary prevention of cardiovascular disease, which is associated with the risk of upper and lower gastro-intestinal tract lesions, particularly in the upper gastro-intestinal tract which may cause asymptomatic lesions to peptic ulcer bleeding and/or even death Li et al. [14].

Until recently, the vitamin $K$ antagonists were the only oral anticoagulant agents available and warfarin remains the most commonly prescribed oral anticoagulation worldwide [15]. Warfarin has significant variability in dose-response across individuals and a narrow therapeutic window and intensive therapeutic monitoring are essential. When combined with low-dose aspirin, NSAIDs, or clopidogrel, warfarin acts cumulatively and the risk of bleeding is significantly increased [16] The risk of major bleeding associated with oral anti-coagulants ranges from 3.26 to $7.2 \%$ annually [11]. Both oral anticoagulation and antiplatelet therapies are essential in $20-30 \%$ of patients with co-existing atrial fibrillation (AF) and $\mathrm{CAD}$, together posing a major risk of thrombotic complications [17]. Currently, in the management of patients with IHD and AF, include triple therapy TT (an anticoagulant plus 2antiplatelet drugs) and two types of dual therapy, DAPT (2 antiplatelet drugs) or DT (an anticoagulant plus a single antiplatelet drug) [18].

\section{Herbal resources and secondary metabolites}

Herbs play an indispensable role in natural product discovery to meet the growing healthcare needs. Researchers screen herbal sources through reverse pharmacology and observational therapeutics to find novel compounds and harness the potential for future drug discovery. According to WHO (World Health Organization), about $80 \%$ of the World's population depends on medicinal plants or herbs to fulfill their medicinal needs. Herbal medicines are a maximum part of complementary and alternative medicine and preferred treatment of people for various reasons such as ethnicity of use, family traditions, and past good experiences [19]. In this review, we have covered 72 herbs, their extracts, their secondary metabolites, and their pharmacological activities studied in both in vivo, ex vivo, and in vitro investigations. Acknowledging the growing significance of traditional medicine and usage, the WHO global report on traditional and complementary medicine 2019 states about the steps taken to promote the safety, quality, and effectiveness of traditional medicine by developing the WHO Traditional Medicine Strategy 2014-2023, in line with WHO Traditional Medicine Strategy (2002-2005). Healthcare professionals need to be aware of and monitor possible risks of concomitant medications of herbs with conventional medicine prescriptions if any [20].

\section{Methods}

We conducted a PubMed search for the in-vitro and in vivo studies published between 2016 and 2020 till December using multiple combinations of keywords, including the following: "anti-thrombotic activity", "antiplatelet activity", "anti-coagulant", "antiplatelet aggregation", "anti-hyper-viscosemia", "anti-aggregant", "platelet agglutination inhibitor", "platelet aggregation inhibitor", "platelet targeted pharmacologic agents", "antiplatelet adhesion", "medicinal plants", and "herbal sources". We found 296 publications that were reviewed by two authors. The retrieved articles were examined 
Table 1 List of herbal sources of antithrombotic and its phytoconstituents

\begin{tabular}{|c|c|c|c|c|c|}
\hline Family & Botanical name & Parts used & Effect/activity & Phytochemicals & References \\
\hline Apiaceae & Angelica keiskei (Miq.) Koidz. & Stem & Antithrombotic-anti-coagulant & Xanthoangelol B & {$[21]$} \\
\hline Apiaceae & Angelica sinensis (Oliv.) Diels & Aerial parts & Anti-coagulant, antiplatelet & Z-Ligustilide & {$[22]$} \\
\hline Malvaceae & Abelmoschus manihot (L.) Medik & Plant & Antiplatelet & Total flavone & {$[23]$} \\
\hline Acanthaceae & $\begin{array}{l}\text { Andrographis paniculata (Burm.f.) } \\
\text { Nees }\end{array}$ & Plant & Antiplatelet & Diterpenoids & {$[24]$} \\
\hline Liliaceae & $\begin{array}{l}\text { Anemarrhena asphodeloides } \\
\text { Bunge }\end{array}$ & Rhizomes & Antiplatelet; antithrombotic & $\begin{array}{l}\text { Timosaponin A-III, timosaponin } \\
\text { B-II, anemarsaponin B, steroidal } \\
\text { glycosides }\end{array}$ & {$[25]$} \\
\hline Apiaceae & Apium graveolens Linn & Seeds & Antithrombotic, antiplatelet & $\begin{array}{l}\text { 3-N-Butylphthalide (NBP)I-3-n- } \\
\text { butylphthalide (NBP) }\end{array}$ & {$[26]$} \\
\hline Amaranthaceae & Achyranthes bidentatata Blume & Plant & Anticoagulant & Polysaccharides & {$[27]$} \\
\hline Liliaceae & Allium sativum $L$. & Cloves & Antiplatelet & $\begin{array}{l}\text { Allicin, adenosine,paraffinic } \\
\text { polysulfides }\end{array}$ & [28] \\
\hline Sapindaceae & Aesculus hippocastanum L. & Bark & Anticoagulant & Aescin (coumarin) & {$[29]$} \\
\hline Berberidaceae & Berberis vulgaris $L$. & Plant & Antiplatelet & Berberine & {$[30]$} \\
\hline Myrtaceae & $\begin{array}{l}\text { Campomanesia xanthocarpa } \\
\text { (Mart.) O.Berg }\end{array}$ & Leaf & Antithrombotic,antiplatelet & Flavonoids & {$[31]$} \\
\hline Cyperaceae & Cyperus rotundus L. & Tuber & Antiplatelet & (+)-nootkatone(sesquiterpenoid) & {$[32]$} \\
\hline Cornaceae & Cornus mas $L$ & Dried fruits & Anticoagulant & Anthocyanins, polyphenols & {$[33]$} \\
\hline Lauraceae & Cassytha filiformis $L$. & Fresh herb & Antiplatelet & Aporphinoid alkaloids & [34] \\
\hline Zingiberaceae & Curcuma aromatica Salisb. & Rhizome & Antiplatelet & Curcumin & {$[35]$} \\
\hline Asteraceae & Chrysanthemum indicum L. & Flowers & Antiplatelet & Chlorogenic acid & {$[36]$} \\
\hline Lauraceae & Cinnamomum cassia Nees. & Bark and twigs & Antiplatelet & $\begin{array}{l}\text { Eugenol, amygdalactone, } \\
\text { cinnamic alcohol, 2-hydroxycin- } \\
\text { namaldehyde, 2-methoxycinna- } \\
\text { maldehyde, coniferaldehyde }\end{array}$ & {$[37]$} \\
\hline Rutaceae & Citrus hassaku Yu.Tanaka & Fruits & Antiplatelet & Prunin & {$[38]$} \\
\hline Ranunculaceae & Coptis chinensis Franch. & Rhizome & Antiplatelet & Berberine & {$[39]$} \\
\hline Compositae & Carthamus tinctorius $L$. & Plant & Antithrombotic & Hydroxysafflor yellow A & {$[40]$} \\
\hline Leguminosae & Caesalpinia sappan L. & Heartwood & Antiplatelet & Brazilin & {$[41]$} \\
\hline Zingiberaceae & Curcuma longa $L$. & Rhizome & $\begin{array}{l}\text { Antiplatelet, anticoagulant, } \\
\text { antithrombotic }\end{array}$ & Ar-turmerone, curcumin & {$[42,43]$} \\
\hline Moraceae & Cudrania tricuspidata Bureau & Roots & Antiplatelet & Cudratricusxanthone A (CTXA) & {$[44]$} \\
\hline Lamiaceae & Callicarpa nudiflora Hook. \& Arn. & Leaves & Antiplatelet & Triterpenoids & {$[45]$} \\
\hline Apiaceae & Centella asiatica L. (Urb). & Herb & Antiplatelet & Caffeoyl quinic acid compounds & {$[46]$} \\
\hline Fabaceae (Leguminosae & Dalbergia odorifera T. Chen & Heartwood & Antiplatelet & Sesquiterpenes & {$[47]$} \\
\hline Dioscoreaceae & Dioscorea zingiberensis C.H. Wright & Rhizome & $\begin{array}{l}\text { Antithrombotic, anticoagulant, } \\
\text { antiplatelet }\end{array}$ & Dioscin-steroidal saponins & {$[48,49]$} \\
\hline Ebenaceae & Diospyros kaki Thunb. & Leaves, fruits & Anticoagulant, antithrombotic & $\begin{array}{l}\text { Diosmin (diosmetin 7-O-rutino- } \\
\text { side), a disaccharide derivative }\end{array}$ & {$[50]$} \\
\hline Euphorbiaceae & Euphorbia neriifolia L. & Roots, leaves & Antithrombotic & Flavonoids, polyphenols & {$[51]$} \\
\hline Rutaceae & Evodia rutaecarpa A.Juss. & Dried unripened fruit & Antiplatelet & Rutaecarpine & {$[52]$} \\
\hline Asteraceae & Erigeron canadensis $L$. & Whole plant & Anticoagulant, antiplatelet & Polyphenolic polysaccharide & [53] \\
\hline Ginkgoaceae & Ginkgo biloba L. & Leaf & Antiplatelet activity & Ginkgolides A, B, and C & {$[54]$} \\
\hline Leguminosae & Glycyrrhiza uralensis & Rhizome & Antithrombotic & |sotrifoliol & {$[55]$} \\
\hline Himantandraceae & Galbulimima baccata F.M.Bailey & Bark & Antithrombotic & Galbulimima alkaloids-himbacine & {$[56]$} \\
\hline Saururaceae & Houttuynia cordata & Plant & Antiplatelet & Alkaloids & {$[57]$} \\
\hline Hernandiaceae & Hernandia nymphaefolia J.Presl. & Trunk bark & Antiplatelet & Aporphine compounds & {$[58]$} \\
\hline Hernandiaceae & Illigera luzonensis Merr & Roots & Antiplatelet & Aporphine alkaloids & {$[59]$} \\
\hline Aquifoliaceae & Ilex paraguariensis A.St. & Fruits & Antithrombotic, antiplatelet & Chikusetsusaponin IVa & {$[60]$} \\
\hline Lamiaceae & Leonurus sibiricus & aerial parts & antiplatelet & Leonurine & {$[61]$} \\
\hline Caprifoliacea & Lonicera japonica Thunb. & plant & antiplatelet & Protocatechuic acid & {$[62]$} \\
\hline Lamiaceaeae & Lycopus lucidus Turcz. & plant & antiplatelet & - & {$[63]$} \\
\hline Asparagaceae & Liriope muscari L.H.Bailey. & plant & anti-thrombotic & D39, a natural saponin & {$[64]$} \\
\hline Lauraceae & Lindera obtusiloba Blume & Leaf & antiplatelet, antithrombotic & quercitrin and afzelin & {$[65]$} \\
\hline Rutaceae & Melicope semecarpifolia Merr. & root bark & antiplatelet & quinoline alkaloids, & {$[66]$} \\
\hline Magnoliaceae & Magnolia officinalis & Bark & antiplatelet & Magnolol,honokiol & {$[67]$} \\
\hline
\end{tabular}


Table 1 (continued)

\begin{tabular}{|c|c|c|c|c|c|}
\hline Family & Botanical name & Parts used & Effect/activity & Phytochemicals & References \\
\hline Nelumbonaceae & Nelumbo nucifera Gaertn. & fruits ; whole plant & anti-coagulant; antithrombotic & $\begin{array}{l}\text { neferine, alkaloid; flavonoids } \\
\text { in hydroalcoholic extract } \\
\text { respectively }\end{array}$ & {$[68]$} \\
\hline Lamiaceae & Origanum majorana L. & plant & antiplatelet & $\begin{array}{l}\text { hydroquinone-D-glucopyrano- } \\
\text { side (Coumarin ) }\end{array}$ & [69] \\
\hline Oleaceae & Osmanthus fragrans Lour. & seeds & antiplatelet & secoiridoid glucoside & [70] \\
\hline Araliaceae & Panax ginseng Meyer & root & antiplatelet & $\begin{array}{l}\text { Ginsenoside Rg1, Ginsenoside } \\
\text { Rg3, Ginsenoside Rp4.Ginse- } \\
\text { noside Ro (an oleanane-type } \\
\text { saponin }\end{array}$ & \\
\hline Piperaceae & Piper longum L. & Dried fruits & antiplatelet & $\begin{array}{l}\text { piperlongumine,a pyridone } \\
\text { alkaloid }\end{array}$ & [71] \\
\hline Paeoniaceae & Paeonia suffruticosa & dried root bark & antiplatelet & - & {$[72]$} \\
\hline Paeoniaceae & Paeonia lactiflora Pall. & plant & antiplatelet and anti-coagulant & $\begin{array}{l}\text { Paeoniflorin, Benzoylpaeoniflorin, } \\
\text { Benzoyloxypaeoniflorin, Methyl } \\
\text { gallate, Catechin, Paeoniflo- } \\
\text { rigenone, Galloylpaeoniflorin, } \\
\text { Daucosterol }\end{array}$ & {$[72]$} \\
\hline Araliaceae & Panax bipinnatifidus Seem. & Roots & antithrombotic,antiplatelet & saponins & [73] \\
\hline Annonaceae & Rollinia mucosa Jaca. & stems & antiplatelet & $\begin{array}{l}\mathrm{N} \text {-methoxycarbonyl aporphine } \\
\text { alkaloids,romucosine } \mathrm{A}(1) \text {, } \\
\text { romucosine } \mathrm{B}(2) \text {, romucosine C } \\
\text { (3), andromucosine D (4 }\end{array}$ & [74] \\
\hline Apocynaceae & Rauwolfia serpentina Benth. & roots & antiplatelet & Ajmaline & [75] \\
\hline Rutacaeae & Ruta graveolens L. & root and aerial parts & antiplatelet & $\begin{array}{l}\text { The quinoline alkaloid graveo- } \\
\text { linine }\end{array}$ & [76] \\
\hline Anacardiaceae & $\begin{array}{l}\text { Rhus verniciflua (Syn.Toxicoden- } \\
\text { dron vernicifluum) }\end{array}$ & herb & antiplatelet & Isomaltol, Pentagalloyl glucose & [77] \\
\hline Polygonaceae & Rheum palmatum $L$. & aerial parts & antiplatelet & $\begin{array}{l}\text { Two stilbenes- trans-resveratrol- } \\
3-O-\beta \text {-d-glucopyranosid (I) and } \\
\text { rhaponticin (II) }\end{array}$ & [78] \\
\hline Scrophulariaceae & Rehmannia glutinosa (Gaertn.) & dried roots & antiplatelet & furan derivatives & [79] \\
\hline Rosaceae & Spiraea japonica L. & roots & antiplatelet & atisine-type diterpenoid alkaloids & {$[80]$} \\
\hline Lamiaceae & Scutellaria baicalensis Georgi. & root & anti-platelet, anticoagulant & Baicalin & {$[81]$} \\
\hline Leguminosae & Spatholobus suberectus Dunn. & stem & antiplatelet & daidzein and genistein & {$[82]$} \\
\hline Fabaceae & Sophora japonica L. & plant & antiplatelet & flavonoids & {$[83]$} \\
\hline Selaginellaceae & $\begin{array}{l}\text { Selaginella tamariscina (P. Beauv.) } \\
\text { Spring }\end{array}$ & herb & anti-coagulant & $\begin{array}{l}\text { dihydrocaffeic acid \& amentof- } \\
\text { lavone }\end{array}$ & {$[84]$} \\
\hline Typhaceae & Sparganium stoloniferum Buch. & plant & antiplatelet, antithrombotic & flavonoids & [9] \\
\hline Labiateae & Salvia miltiorrhiza & Root & antiplatelet & $\begin{array}{l}\text { 15,16-dihydrotanshinone I, } \\
\text { Tanshinone I, Tanshinone IIA, } \\
\text { Cryptotanshinone, Danshensu, } \\
\text { Salvianolic acid B }\end{array}$ & {$[85]$} \\
\hline Sapindaceae & Sapindus mukorossi Gaertn. & Galls & antiplatelet & $\begin{array}{l}\text { Sapinmusaponins F-J; Sapinmu- } \\
\text { saponins Q and R (1-50 } \mu \mathrm{M}) \\
\text { respectively }\end{array}$ & {$[86]$} \\
\hline Asteraceae & Silybum marianum (L.) Gaertn. & Seeds,fruits & antiplatelet activity & Silymarin(flavonolignans) & [87] \\
\hline Rosaceae & Spiraea japonica L. & roots & antiplatelet & spiramine $\mathrm{C} 1$ & {$[80]$} \\
\hline Violaceae & Viola yedoensis Makino & whole plants & anticoagulant & $\begin{array}{l}\text { dicoumarins: dimeresculetin, } \\
\text { euphorbetin, esculetin }\end{array}$ & {$[88]$} \\
\hline Melanthiaceae & $\begin{array}{l}\text { Veratrum dahuricum (Turcz.) } \\
\text { O.Loes. }\end{array}$ & rhizomes & antiplatelet & $\begin{array}{l}\text { Veratroylgermine-steroidal } \\
\text { alkaloid }\end{array}$ & [89] \\
\hline Zingiberaceae & Zingiber officinale Roscoe & rhizome & antiplatelet & Gingerol, paradol & {$[90]$} \\
\hline
\end{tabular}

to eliminate potential duplicates or overlapping data. We also hand-searched the references of relevant articles for the acquisition of additional information. We included only those studies published in peer-reviewed journals in the English language only. Finally, 26 manuscripts were considered for this review. The botanical names of all the plants enumerated below (Table 1) were verified referring to www.theplantlist.org. 


\section{PRISMA FLOWCHART}

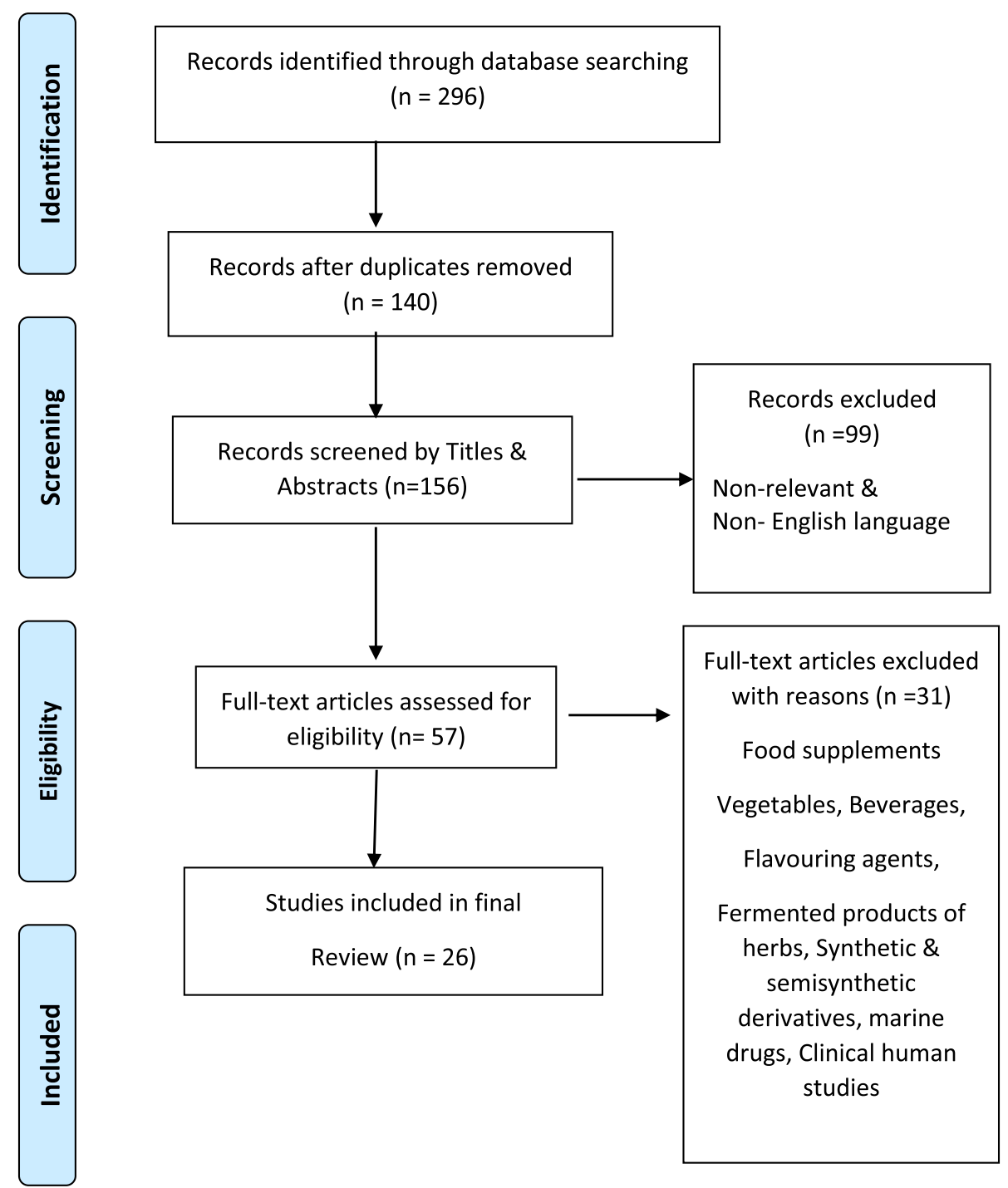

\section{Mechanism of antiplatelet and anticoagulant activity of herbs}

Plant-derived compounds such as alkaloids, anthraquinones, coumarins, flavonoids, xanthones, Lignans, saponins, stilbenes, etc. were found to affect platelet aggregation activity Werner Cordier et al. [91]. Inhibition of platelet adhesion or chemical mediators for activation of platelet function is the common potential of herbs for its antiplatelet activity. Various mechanisms had been postulated such as inhibition of ADP-induced platelet aggregation, inhibition of the arachidonic acid pathway, thereby inhibiting biosynthesis of thromboxane A2; plants containing lignans, xanthones, sesquiterpenes, flavonoids affect coagulation by inhibiting platelet-activating factor (PAF), or PAF receptor antagonists, inhibiting the factor $\mathrm{X}$ on the coagulation cascade. Plants containing the coumarin class of compounds antagonise vitamin $\mathrm{K}$ and 
Table 2 List of herbal sources with mechanisms of its pharmacological action

\begin{tabular}{|c|c|}
\hline Botanical name & Mechanism of action \\
\hline Angelica keiskei (Miq.) Koidz. & Inhibit platelet aggregation \\
\hline Angelica sinensis (Oliv.) Diels & Inhibit platelet aggregation \\
\hline Abelmoschus manihot (L.) Medik & Inhibit platelet aggregation \\
\hline Andrographis paniculata (Burm.f.) Nees & Inhibit platelet aggregation \\
\hline Anemarrhena asphodeloides Bunge & Inhibit ADP-induced platelet aggregation \\
\hline Apium graveolens Linn & Inhibit platelet aggregation \\
\hline Achyranthes bidentatata Blume & Prolonged coagulation time \\
\hline Allium sativum $L$. & Inhibit platelet aggregation \\
\hline Aesculus hippocastanum L. & Preventing oxidative damage of fibrinogen \& moderate antiplatelet aggregation activity \\
\hline Berberis vulgaris $L$. & Inhibit platelet aggregation \\
\hline Campomanesia xanthocarpa (Mart.) O. Berg & Inhibit platelet aggregation, fibrinolytic activity \\
\hline Cyperus rotundus L. & Inhibit collagen-, thrombin-, and AA-induced platelet aggregation \\
\hline Cornus mas L & Inhibit platelet aggregation \\
\hline Cassytha filiformis L. & Inhibit platelet aggregation \\
\hline Curcuma aromatica Salisb. & Inhibit AA-, collagen-, \& ADP-induced platelet aggregation \\
\hline Chrysanthemum indicum $L$. & Inhibit platelet aggregation \\
\hline Cinnamomum cassia Nees. & Inhibit platelet aggregation \\
\hline Citrus hassaku Yu. Tanaka & Inhibit platelet aggregation \\
\hline Coptis chinensis Franch. & Inhibited thromboxane synthesis \\
\hline Carthamus tinctorius $L$. & Inhibited thromboxane synthesis \\
\hline Caesalpinia sappan L. & Inhibited collagen-induced platelet aggregation \\
\hline Curcuma longa $L$. & Inhibit platelet aggregation \\
\hline Cudrania tricuspidata Bureau & Inhibit platelet aggregation, inhibited thrombin production \\
\hline Callicarpa nudiflora Hook. \& Arn. & Antiplatelet aggregation \\
\hline Centella asiatica L. (Urb). & Inhibition of platelet activation and coagulation \\
\hline Dalbergia odorifera T. Chen & Inhibit platelet aggregation \\
\hline Dioscorea zingiberensis C.H. Wright & Antithrombotic \\
\hline Diospyros kaki Thunb. & Inhibited thrombin-catalysed fibrin formation \\
\hline Euphorbia neriifolia L. & Prolonged bleeding time \& clotting time \\
\hline Evodia rutaecarpa A. Juss. & Prolonged bleeding time, antiplatelet aggregation \\
\hline Erigeron canadensis $L$. & Inhibited thrombin \\
\hline Ginkgo biloba L. & Inhibit platelet aggregation \\
\hline Glycyrrhiza uralensis & Antithrombotic \\
\hline Galbulimima baccata F.M. Bailey & Inhibit platelet aggregation \\
\hline Houttuynia cordata & Antiplatelet aggregation \\
\hline Hernandia nymphaefolia J. Presl. & Antiplatelet aggregation \\
\hline Illigera luzonensis Merr & Antiplatelet aggregation \\
\hline Ilex paraguariensis A.St. & Inhibits fibrinogen \& platelet aggregation \\
\hline Leonurus sibiricus & Antiplatelet aggregation \\
\hline Lonicera japonica Thunb. & Antiplatelet aggregation \\
\hline Lycopus lucidus Turcz. & Inhibit aggregation of red blood cells \\
\hline Liriope muscari L.H. Bailey. & Inhibit thrombosis \\
\hline Lindera obtusiloba Blume & Inhibit platelet aggregation \& collagen-induced thromboxane production \\
\hline Melicope semecarpifolia Merr. & Antiplatelet aggregation \\
\hline Magnolia officinalis & Antiplatelet aggregation \\
\hline Nelumbo nucifera Gaertn. & Inhibitory effect on platelet activation, adhesion \& aggregation, and thromboxane A2 formation \\
\hline Origanum majorana L. & Inhibition of platelet adhesion \& aggregation \\
\hline Osmanthus fragrans Lour. & Inhibit platelet aggregation \\
\hline Panax ginseng Meyer & Antiplatelet aggregation \\
\hline
\end{tabular}


Table 2 (continued)

\begin{tabular}{|c|c|}
\hline Botanical name & Mechanism of action \\
\hline Piper longum $L$. & Inhibit AA-, collagen-, \& PAF-induced platelet aggregation \\
\hline Paeonia suffruticosa & Inhibit platelet aggregation \& blood coagulation \\
\hline Paeonia lactiflora Pall. & Inhibit platelet aggregation \& blood coagulation \\
\hline Panax bipinnatifidus Seem. & Inhibit platelet aggregation \& prolonged aPTT \\
\hline Rollinia mucosa Jaca. & Inhibit platelet aggregation \\
\hline Rauwolfia serpentina Benth. & Inhibition of platelet-activating factor \\
\hline Ruta graveolens $L$. & Antiplatelet aggregation \\
\hline Rhus verniciflua (Syn.Toxicodendron vernicifluum) & Antiplatelet aggregation \\
\hline Rheum palmatum $L$. & Antiplatelet aggregation \\
\hline Rehmannia glutinosa (Gaertn.) & Antiplatelet aggregation \\
\hline Spiraea japonica L. & Antiplatelet aggregation \\
\hline Scutellaria baicalensis Georgi. & Inhibited fibrin polymerization and platelet function, prolonged aPTT, PT, and production of thrombin \\
\hline Spatholobus suberectus Dunn. & Inhibition of fibrinogen binding \\
\hline Sophora japonica L. & Antiplatelet aggregation \\
\hline Selaginella tamariscina (P. Beauv.) Spring & Antiplatelet aggregation \& increased fibrinogen content \\
\hline Sparganium stoloniferum Buch. & Antiplatelet aggregation \\
\hline Salvia miltiorrhiza & Inhibit platelet aggregation \\
\hline Sapindus mukorossi Gaertn. & Antiplatelet aggregation \\
\hline \multirow[t]{2}{*}{ Silybum marianum (L.) Gaertn. } & Antiplatelet aggregation \\
\hline & Antiplatelet aggregation \\
\hline Viola yedoensis Makino & Prolonged aPTT, PT \\
\hline Veratrum dahuricum (Turcz.) O. Loes. & Inhibit AA-induced platelet aggregation \\
\hline Zingiber officinale Roscoe & Antiplatelet aggregation \\
\hline
\end{tabular}

$A D P$ adenosine di-phosphate, $A A$ arachidonic acid, $P A F$ platelet-activating factor, aPTT activated partial thromboplastin time, $P T$ prothrombin time

Table 3 Common therapeutic indication of herbs

\begin{tabular}{|c|c|c|}
\hline Herbs & Main uses of herb & Reference \\
\hline Angelica sinensis (Oliv.) Diels & Promoting circulation & Lu et al. [97] \\
\hline Andrographis paniculata (Burm.f.) Nees & Myocardial ischaemia, fever, respiratory infections & Zhang et al. [6] \\
\hline Apium graveolens Linn & Hepatic and spleen disorders, brain disorders, sleep disturbances & Al-Asmari et al. [98] \\
\hline Allium sativum $L$. & Hypercholesterolaemia & Izzo et al. [96] \\
\hline Aesculus hippocastanum L. & Anti-inflammatory, venotonic & Sparg et al. [29] \\
\hline Carthamus tinctorius $L$. & Chest pain, traumatic injuries & Lim et al. [99] \\
\hline Curcuma longa L. & Chest pain, amenorrhoea & Lim et al. [99] \\
\hline Centella asiatica L. (Urb). & Improving memory & Satake et al. [46] \\
\hline Ginkgo biloba L. & CVD, angina, cerebral vasospasm, hypertension & Lim et al. [99] \\
\hline Panax ginseng Meyer & Enhancing immunity, cognitive impairment & Kim et al. [100]; Lim et al. [99] \\
\hline Salvia miltiorrhiza & Cardiovascular and cerebrovascular symptoms & Kim et al. [100] \\
\hline Silybum marianum (L.) Gaertn. & Liver and gallbladder disorders & Gurley et al. [101] \\
\hline Zingiber officinale Roscoe & Anti-bacterial, anti-ulcer & Mohd Nor et al. [102] \\
\hline
\end{tabular}

prevent coagulation. Few naturally occurring compounds contain fibrinolytics which may activate plasminogen and affect coagulation. Phytochemicals that inhibit the CYP3A4, CYP2C9, and CYP1A2 metabolism were potent to affect coagulation Leite et al. [92]. Herbs identified in this review were listed with possible mechanisms of action responsible for their pharmacological activity in Table 2.

\section{Herb-drug interaction types and mechanism}

Among older adults, concomitant herbal medicine use along with prescription drugs had been reported as 5.3 
Table 4 List of herb-aspirin interaction causing increased risk of bleeding

\begin{tabular}{ll}
\hline Botanical name & Herb-aspirin interaction (references) \\
\hline Angelica sinensis (Oliv.) Diels & Xiao et al. [103] \\
Carthamus tinctorius L. & Lim et al. [99] \\
Curcuma longa L. & Hu and Wang [104] \\
Ginkgo biloba L. & Hu and Wang [104] \\
Panax ginseng Meyer & Hu and Wang [104] \\
Salvia miltiorrhiza & Hu and Wang [104]; Xiao et al. [103] \\
\hline
\end{tabular}

Table 5 List of herb-clopidogrel interaction causing increased risk of bleeding

\begin{tabular}{ll}
\hline Botanical name & $\begin{array}{l}\text { Herb-clopidogrel interaction } \\
\text { (references) }\end{array}$ \\
\hline Angelica sinensis (Oliv.) Diels & Xiao et al. [103] \\
Carthamus tinctorius L. & Lim et al. [99] \\
Curcuma longa L. & Lim et al. [99] \\
Ginkgo biloba L. & Lim et al. [99] \\
Panax ginseng Meyer & Lim et al. [99] \\
Salvia miltiorrhiza & Lim et al. [99]; Xiao et al. [103] \\
\hline
\end{tabular}

to $88.3 \%$ in a systematic review as potential cause of herbal-drug interaction Agbabiaka et al. [93]. Herbdrug interactions (HDI) may be either due to pharmacokinetic or pharmacodynamic interactions which affects the safety and efficacy of the treatment. Pharmacokinetic interactions affect the absorption, distribution, metabolism, and excretion of drugs which in turn results in a change in drug concentration in body fluids Lee et al. [94]. Various mechanism has been postulated for the altered drug concentration such as induction or inhibition of hepatic and intestinal drug-metabolizing enzymes such as cytochrome P450, UDP-glucorynyl transferase, and carrier proteins such as P-glycoprotein was suggested Kahrman et al. [95]. While pharmacodynamic interactions are related to the pharmacological activity of the interacting agents which may be synergistic or additive resulting in toxicities or antagonistic causing treatment failure Izzo [96].

\section{Herbal drug interaction with aspirin, clopidogrel, and warfarin}

Few frequently reported herbs, with its commonly used therapeutic indications (Table 3), and drug interactions with conventional anti-thrombotic medicines were enumerated with increased risk of bleeding as per current evidence (Tables 4, 5, and 6) and types of herb-drug interaction of few herbs are summarised (Table 7).

\section{Safety profile}

Salvia miltiorrhiza, Angelica sinensis (Oliv.) Diels and Zingiber officinale Roscoe were identified to cause major interactions with anticoagulant or antiplatelet drugs may lead to life-threatening complications or serious adverse events (Tsai et al. [110]).

\section{Conclusions}

In this review, extensive search has been done on herbal sources investigated for anti-thrombotic activity recently were highlighted. Adverse haemorrhagic complications

Table 6 List of herb-warfarin interaction causing increased risk of bleeding

\begin{tabular}{|c|c|}
\hline Botanical name & Herb-warfarin interaction (references) \\
\hline Angelica sinensis (Oliv.) Diels & Leite et al. [92]; Ge et al. [105]; Akram and Rashid [106]; Leite et al. [107] \\
\hline Andrographis paniculata (Burm.f.) Nees & Leite et al. [107] \\
\hline Apium graveolens Linn & Akram and Rashid [106] \\
\hline Allium sativum $L$. & Leite et al. [92]; Leite et al. [107] \\
\hline Aesculus hippocastanum L. & Leite et al. [107] \\
\hline Carthamus tinctorius $L$. & Leite et al. [107] \\
\hline Curcuma longaL. & Leite et al. [92]; Ge et al. [105]; Akram and Rashid [106]; Shaikh et al. [108]; Leite et al. [107] \\
\hline Centella asiatica L. (Urb). & Leite et al. [107] \\
\hline Ginkgo biloba L. & Leite et al. [92]; Ge et al. [105]; Akram and Rashid [106]; Shaikh et al. [108]; Leite et al. [107] \\
\hline Panax ginseng Meyer & Akram and Rashid [106]; Shaikh et al. [108] \\
\hline Salvia miltiorrhiza & Akram and Rashid [106]; Shaikh et al. [108] \\
\hline Silybum marianum (L.) Gaertn. & Leite et al. [107] \\
\hline Zingiber officinale Roscoe & Leite et al. [92]; Ge et al. [105]; Leite et al. [107] \\
\hline
\end{tabular}


Table 7 Types of herb-drug interaction in herbs

\begin{tabular}{|c|c|c|c|}
\hline Herb & Warfarin & Aspirin & Clopidogrel \\
\hline Angelica sinensis (Oliv.) Diels & $\begin{array}{l}\text { (A) COX-inhibitor [Hu et al. 2005]. Inhib- } \\
\text { its CYP1A2 \& CYP3A4 Leite et al. [92] }\end{array}$ & $\begin{array}{l}\text { (A) Inhibition of rCyp2c1 } 1 \text { \& carboxy- } \\
\text { lesterase activities Xiao et al. [103] }\end{array}$ & $\begin{array}{l}\text { (A) Inhibition of rCyp2c11 \& carboxy- } \\
\text { lesterase activities Xiao et al. [103] }\end{array}$ \\
\hline Allium sativum $L$. & $\begin{array}{l}\text { (A) Intereferes with metabolizing } \\
\text { enzymes Ge et al. [105]; (B) additive } \\
\text { effect [Hu et al. 2005]; (B) PAF inhibitor } \\
\text { Ge et al. [105]; (A) inhibits CYP3A4 Leite } \\
\text { et al. [92] }\end{array}$ & - & - \\
\hline Aesculus hippocastanum L.. & (A) Increased bleeding [Hu et al. 2005] & - & - \\
\hline Carthamus tinctorius $L$. & & (B)Potentiates its activity Lim et al. [99] & $\begin{array}{l}\text { (B) Potentiate prolongation of bleeding } \\
\text { time and prothrombin time Xiao et al. } \\
\text { [103]; (B) potentiates its activity Lim } \\
\text { et al. [99] }\end{array}$ \\
\hline Curcuma longa L. & (B) PAF inhibitor Leite et al. [92] & (A) COX-inhibitor Lim et al. [99] & - \\
\hline Ginkgo biloba L. & $\begin{array}{l}\text { (A) Inhibiting CYP2C9/C19, CYP3A4, } \\
\text { CYP1A2 Costache et al. [109] (B) } \\
\text { Additive effect [Hu et al. 2005]; (B) PAF } \\
\text { receptor antagonist Leite et al. [92] }\end{array}$ & & \\
\hline Panax ginseng Meyer & (B) Additive effect [Hu et al. 2005] & $\begin{array}{l}\text { (B) Inhibited platelet aggregation Lim } \\
\text { et al. [99] }\end{array}$ & \\
\hline Salvia miltiorrhiza & $\begin{array}{l}\text { (A) Increased bleeding; (B) additive } \\
\text { effect [Hu et al. 2005] }\end{array}$ & $\begin{array}{l}\text { (B) Additive or synergistic effect Lim } \\
\text { et al. [99] }\end{array}$ & \\
\hline Zingiber officinale Roscoe & (B) PAF inhibitor Leite et al. [92] & & \\
\hline
\end{tabular}

(A) pharmacokinetic interaction, (B) pharmacodynamic interaction

due to current conventional medicines, patient safety, huge economic burden on healthcare, cognisance of herbal drug interaction, and complications due to recently emerged pandemic due to SARS Co-V2 virus, etc. all pose a need to search for newer pharmacological moieties for drug discovery.

\section{Abbreviations}

CVD: Cardiovascular disease; CAD: Coronary artery disease; PAD: Peripheral arterial disease; VTE: Venous-thrombo-embolic disease; NHANES: The National Health and Nutrition Examination Survey; CHD: Coronary heart disease; AHA: American Heart Association; COVID-19: Coronavirus disease 2019; SARS Co-V2: Severe acute respiratory syndrome coronavirus 2; CAC: Covid-19-associated coagulopathy; ICU: Intensive care unit; ARDS: Acute respiratory distress syndrome; ADR: Adverse drug reaction; NSAID: Non-steroidal anti-inflammatory drug; TT: Triple therapy; DAPT: Dual antiplatelet therapy; DT: Dual therapy; PCl: Percutaneous coronary intervention; IHD: Ischaemic heart disease; AF: Atrial fibrillation; WHO: World Health Organization; HDI: Herb-drug interaction; UDP: Uridine di-phosphate; CYP: Cytochrome.

\section{Acknowledgements}

Authors wish to acknowledge Prof. Dr. K.Kanakavalli, Director General, Central Council for Research in Siddha for encouragement and support.

\section{Authors' contributions}

BS performed conceptualization, review, drafting of manuscript, editing original manuscript. PS contributed conceptualization, review, drafting and editing original manuscript. The author(s) read and approved the final manuscript.

\section{Authors' information}

Dr. Bhavani Subramani is currently working as a Research Officer (Siddha) in Siddha Central Research Institute (SCRI), Central Council for research in Siddha (CCRS), Arumbakkam, Chennai and Dr. P. Sathiyarajeswaran is the Assistant Director \& Incharge, Scientist III, Siddha Central Research Institute (SCRI), Central Council for research in Siddha (CCRS), Arumbakkam, Chennai.

\section{Funding}

No funding.

Availability of data and materials

Data sharing not applicable to this article as no data sets were generated or analyzed during the current study.

\section{Declarations}

Ethics approval and consent to participate

Not applicable.

\section{Consent for publication}

Not applicable.

\section{Competing interests}

Authors have no conflict of interest.

Received: 25 August 2021 Accepted: 1 December 2021 Published online: 07 March 2022

\section{References}

1. Benjamin EJ, Muntner P, Alonso A, Bittencourt MS, Callaway CW, Carson AP et al (2019) Heart disease and stroke statistics-2019 update: a report from the American Heart Association. Circulation. 139(10):e56-e528 Available from: http://ahajournals.org [cited 10 Apr 2021]

2. Virani SS, Alonso A, Benjamin EJ, Bittencourt MS, Callaway CW, Carson AP et al (2020) Heart disease and stroke statistics-2020 update: a report from the American Heart Association. Circulation 141:E139-E596 Available from: http://ahajournals.org Lippincott Williams and Wilkins; [cited 10 Apr 2021] 
3. Singhania N, Bansal S, Nimmatoori DP, Ejaz AA, McCullough PA, Singhania G (2020) Current overview on hypercoagulability in COVID-19. Am J Cardiovasc Drugs. 20(5):393-403 Available from:/pmc/articles/ PMC7398761/ [cited 10 Apr 2021]

4. Klok F, Kruip M, van der Meer N, Arbous M, Gommers D, Kant K et al (2020) Incidence of thrombotic complications in critically ill ICU patients with COVID-19. https://doi.org/10.1016/j.thromres.2020.04.013 [cited 10 Apr 2021]

5. Helms J, Tacquard C, Severac F, Leonard-Lorant I, Ohana M, Delabranche $X$ et al (2020) High risk of thrombosis in patients with severe SARSCoV-2 infection: a multicenter prospective cohort study. Intensive Care Med. 46(6):1089-1098. https://doi.org/10.1007/s00134-020-06062-x [cited 10 Apr 2021]

6. Zhang T, Sun LX, Feng RE (2020) [Comparison of clinical and pathological features between severe acute respiratory syndrome and coronavirus disease 2019]. Zhonghua Jie He He Hu Xi Za Zhi. 43(0):E040. Available from: http://www.ncbi.nlm.nih.gov/pubmed/32241072 [cited 10 Apr 2021]

7. Maiese A, Manetti AC, La Russa R, Di Paolo M, Turillazzi E, Frati P, et al (2020) Autopsy findings in COVID-19-related deaths: a literature review [Internet]. Forensic Science, Medicine, and Pathology. Springer; [cited 10 Apr 2021]. Available from: https://covid19.elsevierpure.com/en/publi cations/autopsy-findings-in-covid-19-related-deaths-a-literature-review

8. Bala MM, Celinska-Lowenhoff M, Szot W, Padjas A, Kaczmarczyk M, Swierz MJ et al (2020) Antiplatelet and anticoagulant agents for secondary prevention of stroke and other thromboembolic events in people with antiphospholipid syndrome. Cochrane Database Syst Rev. 10:CD012169 Available from: https://pubmed.ncbi.nlm.nih.gov/33045766/ [cited 10 Apr 2021]

9. Silvain J, Collet JP, Nagaswami C, Beygui F, Edmondson KE, BellemainAppaix A et al (2011) Composition of coronary thrombus in acute myocardial infarction. J Am Coll Cardiol. 57(12):1359-1367

10. Vine AK (2009) Recent advances in haemostasis and thrombosis. Retina 29(1):1-7 Available from: https://journals.Iww.com/00006982-20090 1000-00001 [cited 10 Apr 2021]

11. Fisher M, Loscalzo J (2011) The perils of combination antithrombotic therapy and potential resolutions. Stroke 42:278-281 Available from: http://stroke.ahajournals.org. Lippincott Williams \& WilkinsHagerstown, MD [cited 10 Apr 2021]

12. De Caterina R, Husted S, Wallentin L, Andreotti F, Arnesen H, Bachmann F et al (2013) General mechanisms of coagulation and targets of anticoagulants (section I): position paper of the ESC Working Group on Thrombosis - Task Force on anticoagulants in heart disease. Thromb Haemost. 109(4):569-579 Available from: https://pubmed.ncbi.nlm.nih. gov/23447024/ [cited 10 Apr 2021]

13. Packard KA, Campbell JA, Knezevich JT, Davis EM (2012) Emerging antiplatelet therapy for coronary artery disease and acute coronary syndrome. Pharmacother J Hum Pharmacol Drug Ther. 32(3):244-273. https://doi.org/10.1002/j.1875-9114.2012.01021.x [cited 10 Apr 2021]

14. Li Z, Wang Z, Shen B, Chen C, Ding X, Song H (2020) Effects of aspirin on the gastrointestinal tract: Pros vs. cons. Oncol Lett 20(3):2567-2578. https://doi.org/10.3892/ol.2020.11817

15. Ageno W, Gallus AS, Wittkowsky A, Crowther M, Hylek EM, Palareti G (2012) Oral anticoagulant therapy - antithrombotic therapy and prevention of thrombosis, 9th ed: American College of Chest Physicians evidence-based clinical practice guidelines. Chest. 141 (2 SUPPL):e44Se88S Available from: /pmc/articles/PMC3278051/ [cited 10 Apr 2021]

16. Hanemaaijer S, Sodihardjo F, Horikx A, Wensing M, De Smet PAGM, Bouvy ML et al (2015) Trends in antithrombotic drug use and adherence to non-vitamin K oral anticoagulants in the Netherlands. Int J Clin Pharm. 37(6):1128-1135. https://doi.org/10.1007/s11096-015-0174-4 [cited 10 Apr 2021]

17. Camm AJ, Kirchhof P, Lip GYH, Schotten U, Savelieva I, Ernst S et al (2010) Guidelines for the management of atrial fibrillation. Eur Heart J 31:2369-2429 Available from: www.escardio.org/guidelines [cited 10 Apr 2021]

18. Zhu W, Guo L, Liu F, Wan R, Shen Y, Lip GYH et al (2017) Efficacy and safety of triple versus dual antithrombotic therapy in atrial fibrillation and ischemic heart disease: a systematic review and meta-analysis. Oncotarget 8:81154-81166 Available from:/pmc/articles/PMC5655270/ Impact Journals LLC; [cited 10 Apr 2021].
19. Welz AN, Emberger-Klein A, Menrad K (2018) Why people use herbal medicine: insights from a focus-group study in Germany. BMC Complement Altern Med. 18(1):92. https://doi.org/10.1186/ s12906-018-2160-6

20. Tsai H-H, Lin H-W, Tsai C-L, Yam FK, Lin S-S (2017) Uncertain associations of major bleeding and concurrent use of antiplatelet agents and Chinese medications: a nested case-crossover study. Evid Based Complement Alternat Med. 2017:9417186 Available from: http://www. ncbi.nlm.nih.gov/pubmed/28831288 [cited 10 Apr 2021]

21. Ohkura N, Nakakuki Y, Taniguchi M, Kanai S, Nakayama A, Ohnishi Ket al (2011) Xanthoangelols isolated from Angelica keiskei inhibit inflammatory-induced plasminogen activator inhibitor 1 (PAl-1) production. BioFactors. 37(6):455-461

22. Zhang L, Du JR, Wang J, Yu DK, Chen YS, He Y et al (2009) Z-ligustilide extracted from Radix angelica sinensis decreased platelet aggregation induced by ADP ex vivo and arterio-venous shunt thrombosis in vivo in rats. Yakugaku Zasshi. 129(7):855-859

23. Fei L, Qianhong W, Yan Y, Haizhen L, Daoheng L, Zhaoping G et al (2020) Traditional uses, chemical constituents, biological properties, clinical settings, and toxicities of Abelmoschus manihot L.: a comprehensive review. Front Pharmacol 11:-1068

24. Thisoda P, Rangkadilok N, Pholphana N, Worasuttayangkurn L, Ruchirawat S, Satayavivad J (2006) Inhibitoryeffect of Andrographis paniculata extract and its active diterpenoids onplatelet aggregation. Eur. J. Pharmacol. 553(1-3):39-45. https://doi.org/10.1016/j.ejphar.2006. 09.052

25. Lu W-Q, Qiu Y, Li T-J, Tao X, Sun L-N, Chen W-S (2011) Antiplatelet and antithrombotic activities of timosaponin B-II, an extract of Anemarrhena asphodeloides. Clin Exp Pharmacol Physiol. 38(7):430-434. https://doi.org/10.1111/j.1440-1681.2011.05530.x [cited 11 Apr 2021]

26. Peng $Y$, Zeng $X$, Feng $Y$, Wang $X$ (2004) Antiplatelet and antithrombotic activity of L-3-n-butylphthalide in rats. J Cardiovasc Pharmacol. 43(6):876-881 Available from: https://pubmed.ncbi.nlm.nih.gov/15167 282/ [cited 3 Apr 2021]

27. He X, Wang X, Fang J, Chang Y, Ning N, Guo H et al (2017) The genus Achyranthes: a review on traditional uses, phytochemistry, and pharmacological activities. J Ethnopharmacol 203:260-278 Elsevier Ireland Ltd

28. Makheja AN, Bailey JM (1990) Antiplatelet constituents of garlic and onion. Agents Actions. 29(3-4):360-363. https://doi.org/10.1007/BF019 66468 [cited 11 Apr 2021]

29. Sparg SG, Light ME, Van Staden J (2004) Biological activities and distribution of plant saponins. J Ethnopharmacol. 94(2-3):219-243

30. Mohd Nor NH, Othman F, Mohd Tohit ER, Md Noor S, Razali R, Ahmad Hassali $\mathrm{H}$ et al (2019) In vitro antiatherothrombotic effects of extracts from Berberis Vulgaris L., Teucrium Polium L., and Orthosiphon Stamineus Benth. Evid Based Complement Altern Med. 2019

31. Klafke JZ, Arnoldi Da Silva M, Fortes Rossato M, Trevisan G, Banderó Walker Cl, Martins Leal CA et al (2012) Antiplatelet, antithrombotic, and fibrinolytic activities of Campomanesia xanthocarpa. Evid Based Complement Altern Med. 2012

32. Seo EJ, Lee DU, Kwak JH, Lee SM, Kim YS, Jung YS (2011) Antiplatelet effects of Cyperus rotundus and its component (+)-nootkatone. J Ethnopharmacol. 135(1):48-54. https://doi.org/10.1016/j.jep.2011.02.025

33. Abdollahi B, Mesgari Abbasi M, Milani PZ, Sadat Nourdadgar A, Banan Khojasteh SM, Nejati V (2014) Hydro-methanolic extract of Cornus mas L. and blood glucose, lipid profile and hematological parameters of male rats. Iran Red Crescent Med J. 16(5)

34. Wu Y, Chang F, Chao Y, Teng C (1998) Antiplatelet and vasorelaxing actions of aporphinoids from Cassytha filiformis. Phyther Res. 12(S1):S39-S41. https://doi.org/10.1002/\%28SICl\%291099-1573\% 281998\%2912\%3A1\%3CS39\%3A\%3AAID-PTR244\%3E3.0.CO\%3B2-O

35. Jantan I, Raweh SM, Sirat HM, Jamil S, Mohd Yasin YH, Jalil J et al (2008) Inhibitory effect of compounds from Zingiberaceae species on human platelet aggregation. Phytomedicine. 15(4):306-309

36. Shao Y, Sun Y, Li D, Chen Y (2020) Chrysanthemum indicum L.: a comprehensive review of its botany, phytochemistry and pharmacology. Am J Chinese Med 48:871-897 World Scientific Publishing Co. Pte Ltd

37. Kim SY, Koo YK, Koo JY, Ngoc TM, Kang SS, Bae K et al (2010) Platelet anti-aggregation activities of compounds from Cinnamomum cassia. J Med Food. 13(5):1069-1074 Available from: https://pubmed.ncbi.nIm. nih.gov/20828311/ [cited 11 Apr 2021] 
38. Itoh K, Masuda M, Naruto S, Murata K, Matsuda H (2010) Effects of unripe Citrus hassaku fruits extract and its flavanone glycosides on blood fluidity. Biol Pharm Bull. 33(4):659-664

39. Xia L-M, Luo M-H (2015) Study progress of berberine for treating cardiovascular disease. Chronic Dis Transl Med. 1(4):231-235. https://doi.org/ 10.1016/j.cdtm.2015.11.006

40. Wu SH, Zheng CP, Chen SY, Cai XP, Shi YJ, Liu Z et al (2014) Anti-thrombotic effect of Carthamus tinctorius linn extracts in rats. Trop J Pharm Res. 13(10):1637-1640

41. Chang Y, Huang SKH, Lu WJ, Chung CL, Chen WL, Lu SH et al (2013) Brazilin isolated from Caesalpinia sappan L. acts as a novel collagen receptor agonist in human platelets. J Biomed Sci. 20:4

42. Kim DC, Ku SK, Bae JS (2012a) Anticoagulant activities of curcumin and its derivative. BMB Rep. 45(4):221-226

43. Srivastava KC, Bordia A, Verma SK (1995) Curcumin, a major component of food spice turmeric (Curcuma longa) inhibits aggregation and alters eicosanoid metabolism in human blood platelets. Prostaglandins Leukot Essent Fatty Acids 52(4):223-227

44. Yoo H, Ku SK, Lee W, Kwak S, Baek YD, Min BW et al (2014) Antiplatelet, anticoagulant, and profibrinolytic activities of cudratricusxanthone A. Arch Pharm Res. 37(8):1069-1078. https://doi.org/10.1007/s12272-0130290-4 [cited 11 Apr 2021]

45. Zhou Z, Wei X, Fu H, Luo Y (2013) Chemical constituents of Callicarpa nudiflora and their anti-platelet aggregation activity. Fitoterapia. 88:91-95

46. Satake T, Kamiya K, An Y, Oishinee Taka T, Yamamoto J (2007) The antithrombotic active constituents from Centella asiatica. Biol Pharm Bull. 30(5):935-940 Available from: http://www.jstage.jst.go.jp/article/bpb/ 30/5/30_5_935/_article [cited 11 Apr 2021]

47. Tao Y, Wang Y (2010) Bioactive sesquiterpenes isolated from the essential oil of Dalbergia odorifera T. Chen. Fitoterapia 81(5):393-396. https:// doi.org/10.1016/j.fitote.2009.11.012

48. Zhang X, Jin M, Tadesse N, Dang J, Zhou T, Zhang H et al (2018a) Dioscorea zingiberensis C. H. Wright: an overview on its traditional use, phytochemistry, pharmacology, clinical applications, quality control, and toxicity. J Ethnopharmacol 220:283-293 Elsevier Ireland Ltd

49. Li H, Huang W, Wen Y, Gong G, Zhao Q, Yu G (2010) Anti-thrombotic activity and chemical characterization of steroidal saponins from Dioscorea zingiberensis C.H. Wright. Fitoterapia. 81(8):1147-1156. https://doi.org/10.1016/j.fitote.2010.07.016

50. You SS, Kim SJ, Choi HS (2005) The anticoagulant fraction from the leaves of Diospyros Kaki L. has an antithrombotic activity. Arch Pharm Res. 28(6):667-674. https://doi.org/10.1007/BF02969356 [cited 11 Apr 2021]

51. Ganeshpurkar A, Hasan M, Bansal D, Dubey N (2014) Protective effect of Euphorbia neriifolia extract on experimentally induced thrombosis in murine model. Niger J Exp Clin Biosci. 2(2):86 Available from: http:// www.njecbonline.org/text.asp?2014/2/2/86/144842 [cited 11 Apr 2021]

52. Sheu JR, Hung WC, Wu CH, Lee YM, Yen MH (2000) Antithrombotic effect of rutaecarpine, an alkaloid isolated from Evodia rutaecarpa, on platelet plug formation in in vivo experiments. $\mathrm{Br} J$ Haematol. 110(1):110-115

53. Pawlaczyk I, Czerchawski L, Kuliczkowski W, Karolko B, Pilecki W, Witkiewicz W et al (2011) Anticoagulant and anti-platelet activity of polyphenolic-polysaccharide preparation isolated from the medicinal plant Erigeron canadensis L. Thromb Res. 127(4):328-340

54. Zuo W, Yan F, Zhang B, Li J, Mei D (2017) Advances in the studies of Ginkgo biloba leaves extract on aging-related diseases. Aging and disease. 8:812-826 Available from: /pmc/articles/PMC5758353/ [cited 11 Apr 2021].

55. Tao WW, Duan JA, Yang NY, Tang YP, Liu MZ, Qian YF (2012) Antithrombotic phenolic compounds from Glycyrrhiza uralensis. Fitoterapia. 83(2):422-425

56. Chackalamannil S, Xia Y (2006) Thrombin receptor (PAR-1) antagonists as novel antithrombotic agents. Exp Opin Ther Pat 16:493-505. https:// doi.org/10.1517/13543776.16.4.493 [cited 11 Apr 2021]

57. Qu W, Wu FH, Li J, Liang JY (2011) Alkaloids from Houttuynia cordata and their antiplatelet aggregation activities. Chin J Nat Med. $9(6): 425-428$

58. Chen JJ, Chang YL, Teng CM, Chen IS (2000) Anti-platelet aggregation alkaloids and lignans from Hernandia nymphaeifolia. Planta Med. 66(3):251-256
59. Chen JJ, Hung HC, Sung PJ, Chen IS, Kuo WL (2011) Aporphine alkaloids and cytotoxic lignans from the roots of Illigera luzonensis. Phytochemistry. 72(6):523-532. https://doi.org/10.1016/j.phytochem.2010.12.015

60. Dahmer T, Berger M, Barlette AG, Reck J, Segalin J, Verza S et al (2012) Antithrombotic effect of chikusetsusaponin IVa isolated from Ilex paraguariensis (Maté). J Med Food. 15(12):1073-1080. https://doi.org/ 10.1089/jmf.2011.0320 [cited 11 Apr 2021]

61. Sayed MA, Alam MA, Islam MS, Ali MT, Ullah ME, Shibly AZ et al (2016) Leonurus sibiricus L. (honeyweed): a review of its phytochemistry and pharmacology. Asian Pac. J Trop Biomed. 6(12):1076-1080. https://doi. org/10.1016/j.apjtb.2016.10.003

62. Kim K, Bae ON, Lim KM, Noh JY, Kang S, Chung KY et al (2012b) Novel antiplatelet activity of protocatechuic acid through the inhibition of high shear stress-induced platelet aggregation. J Pharmacol Exp Ther. 343(3):704-711 Available from: https://jpet.aspetjournals.org/content/ 343/3/704 [cited 11 Apr 2021]

63. Shi HZ, Gao NN, Li YZ, Yu JG, Fan QC, Bai GE et al (2005) Effects of L. F04, the active fraction of lycopus lucidus, on erythrocytes rheological property. Chin J Integr Med. 11(2):132-135

64. Zhai KF, Zheng JR, Tang YM, Li F, LV YN, Zhang YY et al (2017) The saponin D39 blocks dissociation of non-muscular myosin heavy chain IIA from TNF receptor 2, suppressing tissue factor expression and venous thrombosis. Br J Pharmacol. 174(17):2818-2831 Available from: /pmc/ articles/PMC5554322/ [cited 11 Apr 2021].

65. Kim JH, Lee J, Kang S, Moon H, Chung KH, Kim KR (2016) Antiplatelet and antithrombotic effects of the extract of Lindera obtusiloba leaves. Biomol Ther. 24(6):659-664

66. Chen IS, Chen HF, Cheng MJ, Chang YL, Teng CM, Tsutomu I et al (2001) Quinoline alkaloids and other constituents of Melicope semecarpifolia with antiplatelet aggregation activity. J Nat Prod. 64(9):1143-1147. https://doi.org/10.1021/np010122k [cited 11 Apr 2021]

67. Luo H, Wu H, Yu X, Zhang X, Lu Y, Fan J et al (2019) A review of the phytochemistry and pharmacological activities of Magnoliae officinalis cortex. J Ethnopharmacol 236:412-442 Elsevier Ireland Ltd

68. Muhammad Ali Rajput (2019) Assessment of anti-coagulant activity of Nelumbo nucifera fruit - PubMed. Pak J pharm Sci. p. 2561-4. Available from: https://pubmed.ncbi.nlm.nih.gov/31969286/[cited 11 Apr 2021]

69. Yazdanparast $R$, Shahriyary L (2008) Comparative effects of Artemisia dracunculus, Satureja hortensis and Origanum majorana on inhibition of blood platelet adhesion, aggregation and secretion. Vascul Pharmacol. 48(1):32-37

70. Tang W, Cao J, Zhang X, Zhao Y (2015) Osmanthus fragrans seeds, a source of secoiridoid glucosides and its antioxidizing and novel platelet-aggregation inhibiting function. J Funct Foods. 14:337-344

71. Park BS, Son DJ, Park YH, Kim TW, Lee SE (2007) Antiplatelet effects of acidamides isolated from the fruits of Piper longum L. Phytomedicine. 14(12):853-855

72. Yean Kyoung Koo, JMKJYKSSKKBYSKJ-HCHSY-C et al. (2010) Platelet anti-aggregatory and blood anti-coagulant effects of compounds isolated from Paeonia lactiflora and Paeonia suffruticosa - PubMed [Internet]. Pharmazie.. p. 624-8. Available from: https://pubmed.ncbi. nlm.nih.gov/20824965/ [cited 11 Apr 2021]

73. Thom V, Tung N, Van Diep D, Thuy D, Hue N, Long D et al (2018) Antithrombotic activity and saponin composition of the roots of Panax bipinnatifidus Seem. growing in Vietnam. Pharmacognosy Res. 10(4):333 Available from: http://www.phcogres.com/text.asp?2018/10/4/333/ 244092 [cited 11 Apr 2021]

74. Kuo RY, Chang FR, Chen CY, Teng CM, Yen HF, Wu YC (2001) Antiplatelet activity of $\mathrm{N}$-methoxycarbonyl aporphines from Rollinia mucosa. Phytochemistry. 57(3):421-425

75. Rahman NN, Simjee R, Faizi S, Atta-ur-Rahman ASS, Mahmood F et al (1991) Inhibition of platelet activating factor by ajmaline in platelets: in vitro and in vivo studies. Pak J Pharm Sci. 4(1):35-42 Available from: http://www.ncbi.nlm.nih.gov/pubmed/16414679 [cited 11 Apr 2021]

76. Wu T-S, Shi L-S, Wang J-J, lou S-C, Chang H-C, Chen Y-P et al (2003) Cytotoxic and antiplatelet aggregation principles of Ruta Graveolens. J Chinese Chem Soc. 50(1):171-178. https://doi.org/10.1002/jccs.20030 0024 [cited 11 Apr 2021]

77. Jeon WK, Lee JH, Kim HK, Lee AY, Lee SO, Kim YS et al (2006) Antiplatelet effects of bioactive compounds isolated from the bark of Rhus verniciflua Stokes. J Ethnopharmacol. 106(1):62-69 
78. Aburjai TA (2000) Anti-platelet stilbenes from aerial parts of Rheum palaestinum. Phytochemistry. 55(5):407-410

79. Sen LY, Chen ZJ, Zhu DY (2005) A novel bis-furan derivative, two new natural furan derivatives from Rehmannia glutinosa and their bioactivity. Nat Prod Res 19(2):165-170. https://doi.org/10.1080/14786410410001704787

80. Li L, Shen YM, Yang XS, Zuo GY, Shen ZQ, Chen ZH et al (2002) Antiplatelet aggregation activity of diterpene alkaloids from Spiraea japonica. Eur J Pharmacol. 449(1-2):23-28

81. Lee W, Ku SK, Bae JS (2015) Antiplatelet, anticoagulant, and profibrinolytic activities of baicalin. Arch Pharm Res. 38(5):893-903. https://doi. org/10.1007/s12272-014-0410-9 [cited 11 Apr 2021]

82. Lee BJ, Jo IY, Bu Y, Park JW, Maeng S, Kang H et al (2011) Antiplatelet effects of Spatholobus suberectus via inhibition of the glycoprotein Ilb/ Illa receptor. J Ethnopharmacol. 134(2):460-467

83. Kim JM, Yun-Choi HS (2008) Anti-platelet effects of flavonoids and flavonoid-glycosides from Sophora japonica. Arch Pharm Res. 31(7):886-890. https://doi.org/10.1007/s12272-001-1242-1 [cited 11 Apr 2021]

84. Zhang Q, Wang YL, Gao D, Cai L, Yang YY, Hu YJ et al (2018b) Comparing coagulation activity of Selaginella tamariscina before and after stirfrying process and determining the possible active constituents based on compositional variation. Pharm Biol. 56(1):67-75. https://doi.org/10. 1080/13880209.2017.1421673

85. Park JW, Lee SH, Yang MK, Lee JJ, Song MJ, Ryu SY et al (2008) 15,16-Dihydrotanshinone I, a major component from Salvia miltiorrhiza Bunge (Dansham), inhibits rabbit platelet aggregation by suppressing intracellular calcium mobilization. Arch Pharm Res. 31(1):47-53. https:// doi.org/10.1007/s12272-008-1119-4 [cited 11 Apr 2021]

86. Huang HC, Tsai WJ, Morris-Natschke SL, Tokuda H, Lee KH, Wu YC et al (2006) Sapinmusaponins F-J, bioactive tirucallane-type saponins from the galls of Sapindus mukorossi. J Nat Prod. 69(5):763-767. https://doi. org/10.1021/np050446z [cited 11 Apr 2021]

87. Pourová J, Applová L, Macáková K, Vopršalová M, Migkos T, Bentanachs R et al (2019) The effect of silymarin flavonolignans and their sulfated conjugates on platelet aggregation and blood vessels ex vivo. Nutrients. 11(10)

88. Zhou HY, Hong JL, Shu P, Ni YJ, Qin MJ (2009) A new dicoumarin and anticoagulant activity from Viola yedoensis Makino. Fitoterapia. 80(5):283-285 Available from: https://pubmed.ncbi.nlm.nih.gov/19306 914/ [cited 11 Apr 2021]

89. Tang J, Li H-L, Shen Y-H, Jin H-Z, Yan S-K, Liu X-H et al (2010) Antitumor and antiplatelet activity of alkaloids from veratrum dahuricum. Phyther Res. 24(6):821-826. https://doi.org/10.1002/ptr.3022 [cited 11 Apr 2021]

90. Liao YR, Leu YL, Chan YY, Kuo PC, Wu TS (2012) Anti-platelet aggregation and vasorelaxing effects of the constituents of the rhizomes of zingiber officinale. Molecules. 17(8):8928-8937

91. Cordier W, Steenkamp V (2012) Herbal remedies affecting coagulation: A review. Pharm Biol 50(4):443-452. https://doi.org/10.3109/13880209. 2011.611145

92. Leite PM, Martins M, Castilho RO (2016) Review on mechanisms and interactions in concomitant use of herbs and warfarin therapy. Biomed Pharmacother 83:14-21. https://doi.org/10.1016/j.biopha.2016.06.012

93. Agbabiaka TB, Wider B, Watson LK, Goodman C (2017) Concurrent Use of Prescription Drugs and Herbal Medicinal Products in Older Adults: A Systematic Review. Drugs Aging 34(12):891-905. https://doi.org/10. 1007/s40266-017-0501-7

94. Lee W, Lee S, Choi J, et al (2017) Antithrombotic properties of JJ1, a potent and novel thrombin inhibitor. Sci Rep 14862. https://doi.org/10. 1038/s41598-017-13868-1

95. Kahrman C, Arituluk ZC, Cankaya IIT (2020). The Clinical importance of herb-drug interactions and toxicological risks of plants and herbal products. https://doi.org/10.5772/intechopen.92040.

96. Izzo AA (2012) Interactions between herbs and conventional drugs: overview of the clinical data. Med Princ Pract: international journal of the Kuwait University, Health Science Centre 21(5):404-428. https://doi. org/10.1159/000334488

97. Lu C, Liu M, Shang W, Yuan Y, Li M, Deng X, Li H, Yang K (2020) Knowledge Mapping of Angelica sinensis (Oliv.) Diels (Danggui) Research: A Scientometric Study. Front Pharmacol 11:294. https://doi.org/10.3389/ fphar.2020.00294

98. Al-Asmari AK, Athar MT, Kadasah SG (2017) An Updated Phytopharmacological Review on Medicinal Plant of Arab Region: Apium graveolens
Linn. Pharmacogn Rev 11(21):13-18. https://doi.org/10.4103/phrev. phrev_35_16

99. Lim JW, Chee SX, Wong WJ, He QL, Lau TC (2018) Traditional Chinese medicine: herb-drug interactions with aspirin. Singapore Med J 59(5):230-239. https://doi.org/10.11622/smedj.2018051

100. Kim K, Park KI (2019) A Review of Antiplatelet Activity of Traditional Medicinal Herbs on Integrative Medicine Studies. Evidence-based complementary and alternative medicine. eCAM 7125162. https://doi. org/10.1155/2019/7125162

101. Gurley BJ, Tonsing-Carter A, Thomas SL, Fifer EK (2018) Clinically Relevant Herb-Micronutrient Interactions: When Botanicals, Minerals, and Vitamins Collide. Adv Nutr 9(4):524S-532S. https://doi.org/10.1093/ advances/nmy029

102. Mohd Nor NH, Othman F, Mohd Tohit ER, Md Noor S (2016) Medicinal Herbals with Antiplatelet Properties Benefit in Coronary Atherothrombotic Diseases. Thrombosis 5952910. https://doi.org/10.1155/2016/5952910

103. Xiao M, Qian C, Luo X, Yang M, Zhang Y, Wu C, Mok C, Lee P, Zuo Z (2019) Impact of the Chinese herbal medicines on dual antiplatelet therapy with clopidogrel and aspirin: Pharmacokinetics and pharmacodynamics outcomes and related mechanisms in rats. J Ethnopharmacol 235:100-110. https://doi.org/10.1016/j.jep.2019.01.040

104. Hu Y, Wang J (2019) Interactions between clopidogrel and traditional Chinese medicine. J Thromb Thrombolysis 48(3):491-499. https://doi. org/10.1007/s11239-019-01945-3

105. Ge B, Zhang Z, Zuo Z (2014) Updates on the clinical evidenced herbwarfarin interactions. Evidence-based complementary and alternative medicine: eCAM 957362. https://doi.org/10.1155/2014/957362

106. Akram M, Rashid A (2017) Anti-coagulant activity of plants: mini review. J Thromb Thrombolysis 44(3):406-411. https://doi.org/10.1007/ s11239-017-1546-5

107. Leite PM, Parreiras Martins MA, das Graças Carvalho M, Oliveira Castilho R (2021) Mechanisms and interactions in concomitant use of herbs and war far in therapy: An updated review. Biomed Pharmacother 143:112103, ISSN 0753-3322. https://doi.org/10.1016/j.biopha.2021.112103. (https:// www.sciencedirect.com/science/article/pii/S0753332221008878)

108. Shaikh AS, Thomas AB, Chitlange SS (2020) Herb-drug interaction studies of herbs used in treatment of cardiovascular disorders-A narrative review of preclinical and clinical studies. Phytother Res 34(5):10081026. https://doi.org/10.1002/ptr.6585

109. Costache I-I, Miron A, Hăncianu M, Aursulesei V, Dan Costache A, Aprotosoaie AC (2019) Pharmacokinetic Interactions between Cardiovascular Medicines and Plant Products. Cardiovasc Ther 9402781:19. https:// doi.org/10.1155/2019/9402781

110. Tsai HH, Lin HW, Lu YH, Chen YL, Mahady GB (2013) A review of potential harmful interactions between anticoagulant/antiplatelet agents and Chinese herbal medicines. PloS one 8(5):e64255. https://doi.org/10. 1371/journal.pone.0064255

\section{Publisher's Note}

Springer Nature remains neutral with regard to jurisdictional claims in published maps and institutional affiliations.

\section{Submit your manuscript to a SpringerOpen ${ }^{\circ}$ journal and benefit from:}

- Convenient online submission

- Rigorous peer review

- Open access: articles freely available online

- High visibility within the field

Retaining the copyright to your article

Submit your next manuscript at springeropen.com 